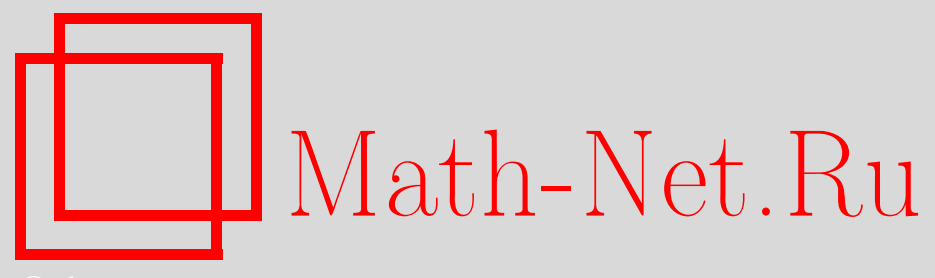

А. Блинков, Равновеликость от Произволова, Квант, 2020, номер 9, 29-33

DOI: https://doi.org/10.4213/kvant20200904

Использование Общероссийского математического портала Math-Net.Ru подразумевает, что вы прочитали и согласны с пользовательским соглашением http://www.mathnet.ru/rus/agreement

Параметры загрузки:

IP : 35.173 .219 .149

26 апреля 2023 г., $14: 34: 00$

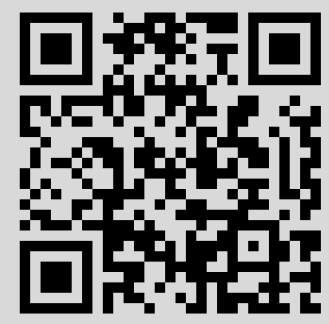




\section{Равновеликость от Произволова}

\section{А.БЛИНКОВ}

\section{$\mathrm{B}$} ЯЧЕСЛАВ ВИКТОРОВИЧ ПРОИЗволов был выдающимся композитором математических задач для школьников. Многие из них вошли в его замечательную книжку «Задачи на вырост», изданную последний раз в 2003 году, но далеко не все. Сейчас в МЦНМО готовится новое, дополненное издание этой книги.

Большинство задач В.В.Произволова отличают запоминающиеся факты в условиях, умение по-новому преподнести, казалось бы, известную идею, краткость и изящество решений. Будучи настоящим математиком, «поймав» какую-либо идею, он старался ее развить, поэтому его задачи легко объединять в серии, из которых можно составлять кружковые занятия для школьников, что и делают многие преподаватели.

Одна из серий задач Произволова уже была представлена в статье «Угол в квадрате» («Квант» №4 за 2014 г.). А для этой статьи отобраны задачи, связанные с площадями. В них можно проследить несколько излюбленных идей и приемов Вячеслава Викторовича. Подчеркнем, что многие из предлагаемых задач можно решить разными способами, но мы будем обсуждать именно авторские решения, которые не требуют громоздких вычислений и в то же время, как правило, содержат глубокие идеи.

Начнем со сравнительно простой задачи, идея решения которой известна практически каждому школьнику, но ее не так-то просто опознать.

Задача 1. Параллелограммы $A B C D$ u BEFG с общей вершиной $B$ расположень так, что точка $C$ лежит на отрезке $E F, a$ точка $G$ - на отрезке AD. Докажите, что эти параллелограммы равновелики.

Решение. Площадь каждого параллелограмма равна удвоенной площади треугольника $B C G$, так как этот треугольник имеет

DOI: https://doi.org/10.4213/kvant20200904 общее основание и равную высоту с каждым из параллелограммов (рис.1).

А вот похожая идея, но ее увидеть и реализовать существенно

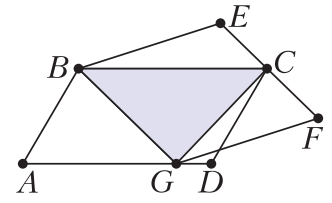

РИс. 1 сложнее.

Задача 2. На стороне АC треугольника $A B C$ задана точка $B_{1}$. На сторонах $A B$ и $B C$ постройте точки $C_{1}$ и $A_{1}$ соответственно так, чтобы площади треугольников $A B_{1} C_{1}$, $B C_{1} A_{1}$ и $C A_{1} B_{1}$ были равны.

Решение. Равновеликость (равенство площадей) указанных треугольников равносильна равновеликости параллелограммов, для которых стороны треугольника $A_{1} B_{1} C_{1}$ являются диагоналями (рис.2).

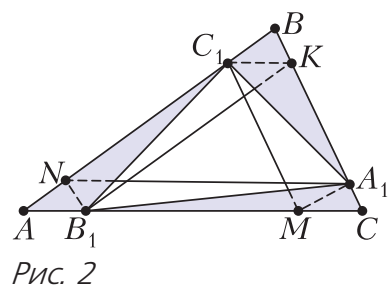

Отсюда следует, что надо построить ломаную $B_{1} K C_{1} M A_{1} N$, последовательные звенья которой соответственно параллельны $A B$, $A C, B C, A B$ и $A C$. Тогда площадь параллелограмма $A C_{1} K B_{1}$ равна площади параллелограмма $C K C_{1} M$ (у них общая сторона $C K$ и равны высоты, проведенные к ней), а площадь $C K C_{1} M$, в свою очередь, равна площади параллелограмма $B C_{1} M A_{1}$ (у них общая сторона $C_{1} M$ и равны высоты, проведенные к ней). Из равновеликости $A C_{1} K B_{1}$ и $B C_{1} M A_{1}$ следует равенство площадей треугольников $A B_{1} C_{1}$ и $B C_{1} A_{1}$.

Чтобы доказать, что треугольник $C A_{1} B_{1}$ имеет такую же площадь, достаточно провести аналогичное рассуждение. Но сначала надо доказать, что $C B_{1} N A_{1}$ - параллелограмм. Это несложно, так как $N A_{1} \| B_{1} C$ по построению, а равенство этих сторон следует из того, что $A B_{1}=C_{1} K=M C$.

В следующей задаче - другой случай стандартной равновеликости (и ее надо сначала увидеть!). Но прежде всего она служит иллюстрацией еще одной идеи, которая привлекала Произволова, - это использование объединения и пересечения фигур. 
a)

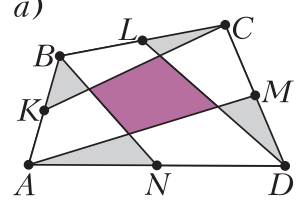

Pис. 3

Задача 3. Точки K, L, M и N-середины сторон выпуклого четырехугольника $A B C D$ (рис.3,a). Докажите, что сумма площадей закрашенных треугольников равна площади закрашенного четырехугольника.

Решение. Проведем в данном четырехугольнике диагональ $A C$ (рис.3,б). Так как $C K$ - медиана треугольника $A B C$, то $S_{B C K}=$ $=\frac{1}{2} S_{A B C}$. Аналогично, $S_{A M D}=\frac{1}{2} S_{A C D}$, значит, $S_{B C K}+S_{A M D}=\frac{1}{2} S_{A B C D}$. Проведя диагональ $B D$, аналогичными рассуждениями получим, что $S_{A B N}+S_{C L D}=\frac{1}{2} S_{A B C D}$. Таким образом, сумма площадей четырех треугольников, указанных выше, равна площади $A B C D$. Следовательно, та часть площади $A B C D$, которую они покрывают дважды, т.е. сумма площадей закрашенных треугольников, равна той части площади $A B C D$, которую они вообще не покрывают (площадь закрашенного четырехугольника).

Во многих задачах В.В.Произволова требуется сравнить площади частей фигуры, по-разному закрашенных. Вот пример, в котором, наряду с применением пересечения, используется идея перекладывания частей.

Задача 4. Правильный восьмиугольник разрезан на части, которые закрашены в четыре ивета ( рис.4). Докажите, что каждым иветом закрашена одна и та же пло$u_{\imath} a \partial$.

Решение. Поменяв местами равные треугольники: лиловый и

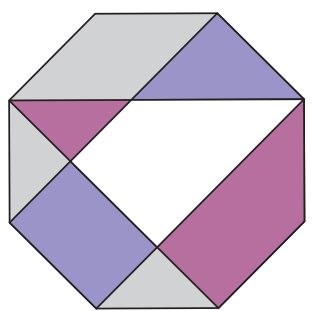
нижний серый, полу- Рис. 4 чим две одинаковые равнобокие трапеции, у которых меньшее основание равно ее боковой стороне, а угол при большем основании равен $45^{\circ}$.
Кроме того, если разрезать сиреневый прямоугольный треугольник на два треугольника, проведя высоту к гипотенузе, и заменить этими треугольниками два серых, то получится такая же трапеция.

Площадь восьмиугольника равна сумме площадей четырех таких трапеций, так как из четырех прямоугольных треугольников их попарного пересечения можно сложить центральный квадрат, дополняющий трапеции до восьмиугольника. Следовательно, белая часть имеет такую же площадь.

А вот та же идея перекладывания частей, но в более сложном исполнении.

Задача 5. $B$ выпуклом шестиугольнике $A C_{1} B A_{1} C B_{1}: A B_{1}=A C_{1}, B C_{1}=B A_{1}, C A_{1}=C B_{1}$ $u \angle A+\angle B+\angle C=\angle A_{1}+\angle B_{1}+\angle C_{1}$. Докажите, что площадь треугольника АВС равна половине площади шестиугольника.

Решение. Отрежем от шестиугольника треугольники $B A_{1} C, C B_{1} A, A C_{1} B$ и приложим их друг к другу так, чтобы вершины $A_{1}, B_{1}$ и $C_{1}$ совместились в некоторой точке $O$, сторона $A_{1} C$ первого треугольника совместилась со стороной $B_{1} C$ второго, а сторона $B_{1} A$ второго - со стороной $C_{1} A$ третьего (рис.5).

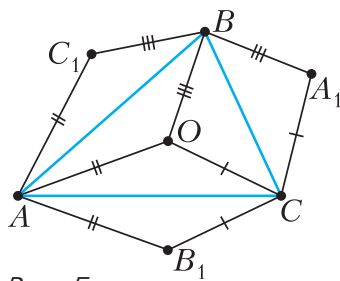

Pис. 5
Так как сумма углов шестиугольника равна $720^{\circ}$, то $\angle A_{1}+\angle B_{1}+\angle C_{1}=360^{\circ}$. Отсюда следует, что сторона $C_{1} B$ третьего треугольника автоматически совместится со стороной $A_{1} B$ первого. Таким образом, из трех отрезанных треугольников мы сложили треугольник со сторонами, равными $B C, C A$ и $A B$, поэтому он равен треугольнику $A B C$. Следовательно, $S_{B A_{1} C}+S_{C B_{1} A}+S_{A C_{1} B}=S_{A B C}$, значит, площадь треугольника $A B C$ равна половине площади шестиугольника.

Из доказанного следует, что точка $O$ симметрична вершинам $A_{1}, B_{1}$ и $C_{1}$ относительно соответствующих сторон треугольника $A B C$, поэтому описанное построение равносильно свертыванию треугольников $B A_{1} C, C B_{1} A$, $A C_{1} B$ внутрь шестиугольника по отрезкам $B C, C A$ и $A B$ соответственно. 
Вот другой пример использования этой же идеи.

Задача 6. Вокруг прямоугольника описан четырехугольник так, что две противоположные вершины прямоугольника являются серединами двух противоположных сторон четырехугольника. Докажите, что площадь прямоугольника равна половине площади четырехугольника.

Решение. Пусть $K L M N$ - данный прямоугольник, $A B C D$ - описанный около него четырехугольник, $K$ - середина $A B, M$ - середина $C D$ (рис.6, $a$ ). Перегнем внутрь прямоугольника четыре треугольника: $K A N, K B L$, $M C L$ и $M D N$. Так как $K A=K B$ и $\angle A K N+$ $+\angle B K L=90^{\circ}=\angle L K N$, то вершины $A$ и $B$ совместятся в точке $E$. Аналогично, вершины

a)

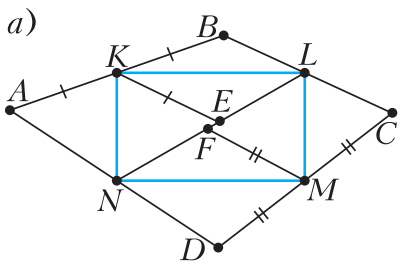

Рис. 6

$C$ и $D$ совместятся в точке $F$. Кроме того, точки $E$ и $F$ окажутся на одной прямой как с вершиной $L$, так и с вершиной $N$. Значит, если $E$ и $F$ не совпадают, то они лежат на $L N$. Возможен и случай совпадения точек $E$ и $F$ (рис.6,б), но в обоих случаях указанные треугольники накрывают прямоугольник без просветов и перекрытий, поэтому площадь $K L M N$ равна половине площади $A B C D$.

Идею свертывания В.В.Произволов использовал в разных задачах, не только на площади. Она связана с идеей использования осевых симметрий и их композиции. Поэтому перейдем к серии задач, в которых напрямую или завуалированно использованы различные движения на плоскости.

Задача 7. Из бумажного прямоугольника вырезали два одинаковых круга. Проведите прямую, деляшую получившуюся фигуру на две части равной площади.

Решение. Любая прямая, проходящая через центр симметрии прямоугольника, делит его на две равные части, которые имеют равные площади. Для того чтобы вырезанные части в обеих фигурах имели равные площади, искомая прямая $m$ должна также

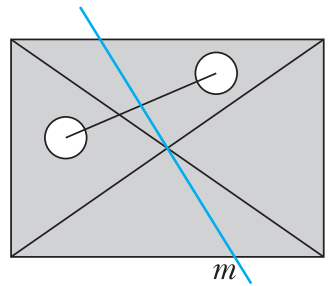

Pnc. 7 проходить через центр симметрии вырезанных кругов (рис.7).

В ситуации, показанной на рисунке 7 , достаточно, чтобы прямая прошла через центр прямоугольни-

ка, а вырезанные круги оказались в разных полуплоскостях относительно нее. Однако требование, чтобы прямая проходила через центр симметрии двух вырезанных кругов, становится принципиальным, если, например, один из вырезанных кругов содержит центр прямоугольника.

Задача 8. $B$ выпуклом пятиугольнике $A B C D E: \quad B C=C D=A E=1, \quad A B+D E=1$, $\angle A B C=\angle C D E=90^{\circ}$. Hайдите его плошадь.

Решение. «Отрежем» треугольник $A B C$ и «приложим» его так, чтобы сторона $B C$ совместилась со стороной $D C$, при этом точка $A$ займет положение $A^{\prime}$ (рис.8). Таккак $\angle A B C=$ $=\angle C D E=90^{\circ}$, то точки $E, D$ и $A^{\prime}$ будут лежать на одной пря-

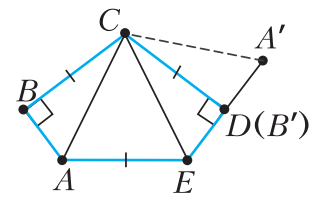

Pис. 8 мой, значит, $E A^{\prime}=A^{\prime} B^{\prime}+D E=1$. Следовательно, $S_{A^{\prime} C E}=0,5$. Но треугольник $A^{\prime} C E$ равен треугольнику $A C E$ (по трем сторонам), поэтому $S_{A B C D E}=S_{A C A^{\prime} E}=1$.

По сути, был выполнен поворот треугольника $A B C$ с центром $C$ на угол $C B D$ против часовой стрелки.

Задача 9. На стороне AD параллелограмма $A B C D$ отмечена точка $M$, а на сторонах $A B$ и $C D$ - такие точки $P$ и $Q$, что $P M\|B D, Q M\| A C$. Докажите, что равны площади треугольников РMB и QMC.

Решение. Заметим, что точки $P$ и $Q$ симметричны относительно точки $O$ пересечения диагоналей параллелограмма (рис.9). Действительно, по теореме о пропорцио-

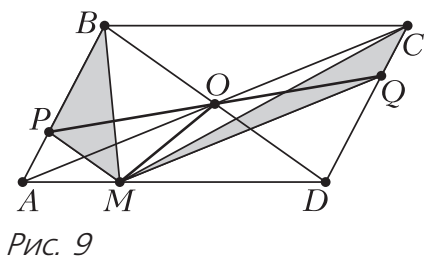


нальных отрезках $\frac{A P}{A B}=\frac{A M}{A D}=\frac{C Q}{C D}$, откуда $A P=C Q$. Проведем отрезки $P Q$ и $O M$. Так как $B P M O$ - трапеция, то $S_{P M B}=S_{M P O}$. Аналогично, из трапеции $M O C Q$ получим, что $S_{Q M C}=S_{M Q O}$. Но $M O$ - медиана треугольника $P M Q$, поэтому $S_{M P O}=S_{M Q O}$, следовательно, $S_{P M B}=S_{Q M C}$, что и требовалось.

Задача 10. Угол В при вершине равнобедренного треугольника АВС равен $120^{\circ}$. Из вершины B выпустили внутрь треугольни$\kappa а ~ д в а ~ л у ч а ~ п о д ~ у г л о м ~ 60^{\circ}$ друг к другу, которые, отразившись от основания АС в точках $P$ и $Q$, попали на боковые стороны в точки М и N (рис.10,a). Докажите, что площадь треугольника $P B Q$ равна сумме площадей треугольников АМP и CNQ.
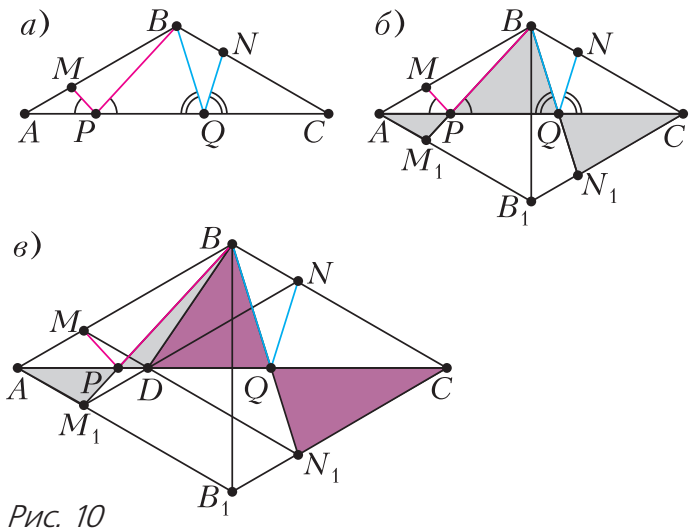

Решение. Отразим картинку относительно прямой $A C$, обозначив точки, симметричные $M, B$ и $N$, через $M_{1}, B_{1}$ и $N_{1}$ соответственно (рис. $10, \sigma, в)$. Заметим. что треугольники $A B M_{1}$ и $B_{1} B N_{1}$ равны (один получается из другого поворотом на $60^{\circ}$ вокруг точки $B$ ). Учитывая симметрию, достаточно доказать, что $S_{B P Q}=S_{A P M_{1}}+S_{C Q N_{1}}$. Далее можно рассуждать по-разному.

Первый способ. Добавив к обеим частям этого равенства $S_{M_{1} P Q N_{1} B_{1}}$, сведем задачу к доказательству равенства $S_{A C B_{1}}=S_{B M_{1} B_{1} N_{1}}$ (см. рис.10,6). По доказанному выше $S_{B M_{1} B_{1} N_{1}}=S_{M_{1} B B_{1}}+S_{B_{1} B N_{1}}=S_{M_{1} B B_{1}}+S_{A B M_{1}}=$ $=S_{A B B_{1}}=S_{A C B_{1}}$.

Второй способ. Из симметрии и поворота следует, что $M B=M_{1} B_{1}=N_{1} C$. Проведем отрезок $M N_{1}$, пересекающий $A C$ в точке $D$ (см. рис.10,в). В силу симметрии отрезок $N M_{1}$ пройдет через ту же точку $D$. Так как отрезки $M B$ и $N_{1} C$ параллельны и равны, то $M B C N_{1}$ - параллелограмм, а $B D N_{1} C$ - трапеция. Значит, $S_{B D Q}=S_{C N_{1} Q}$. Аналогично, из трапеции $B D M_{1} A$ получим, что $S_{B D P}=$ $=S_{A M_{1} P}$. Значит, $S_{B P Q}=S_{A P M_{1}}+S_{C Q N_{1}}$.

\section{Задачи для самостоятельного решения}

Эти задачи содержат те же идеи, причем большинство из них можно назвать либо «предками», либо «потомками» уже разобранных задач.

11. Из бумажного прямоугольника вырезали прямоугольник. Проведите прямую, делящую получившуюся фигуру на две части равной площади.

12. В четырехугольнике $A B C D: A B=B C$, $\angle A B C=\angle A D C=90^{\circ}$. Найдите его площадь, если расстояние от вершины $B$ до прямой $A D$ равно 1 .

13. Прямоугольный лист бумаги разрезали на три треугольника. Сумма площадей двух из них в два раза больше площади третьего. Найдите отношение площадей этих треугольников.

14. Противолежащие стороны шестиугольника $A B C D E F$ равны и параллельны. Найдите площадь треугольника $A C E$, если площадь шестиугольника равна $S$.

15. Внутри равностороннего треугольника отмечена точка, которая соединена отрезками с вершинами, и из нее проведены перпендикуляры к сторонам (рис.11). Три из образовавшихся шести треугольников закрашены (через один). Докажите, что сумма площадей закрашенных треугольников равна половине площади данного треугольника.

16. Какая часть квадрата $A B C D$ имеет большую площадь: серая или лиловая (рис.12)?

17. Две диагонали разбили правильный восьмиугольник на две трапеции и прямоугольник. Докажите, что площадь прямоугольника равна половине площади восьмиугольника.

18. Правильный девятиугольник разрезан диагоналями на белые и закрашенные треугольники

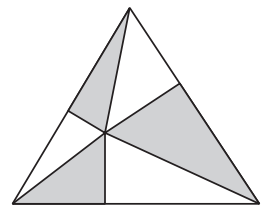

Pис. 11

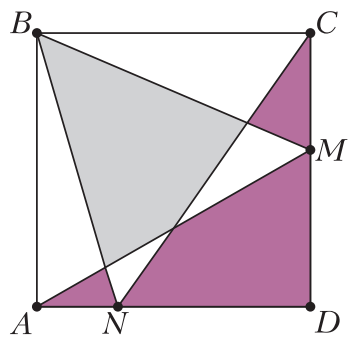

Pис. 12 


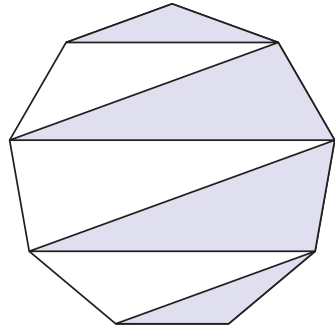

Рис. 13

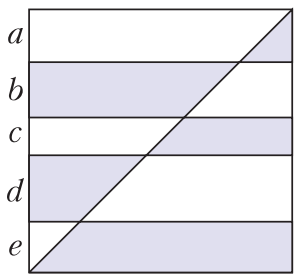

Рис. 14

(рис.13). Какая часть площади больше: белая или закрашенная?

19. Квадрат разрезан на полоски, ширина которых обозначена буквами $a, b, c, d, e$, проведена его диагональ и полученные части закрашены так, как показано на рисунке 14. Известно, что $a+c+e=b+d$. Докажите, что равны суммы площадей закрашенных частей, расположенных выше и ниже диагонали.

20. Прямоугольники $A B C D$ и $K L M N$ с соответственно параллельными сторонами расположены так, как показано на рисунке 15. Докажите равенство площадей четырехугольников $A L C N$ и KBMD.

21. На стороне $A B$ параллелограмма $A B C D$ отмечена точка $P$. Постройте параллелограмм с вершиной $P$, вписанный в $A B C D$ так, что его

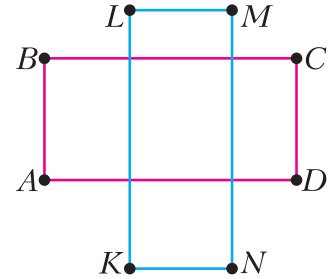

Рис. 15

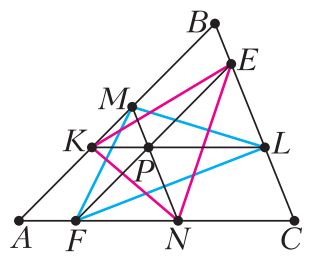

Pис. 16

стороны отсекают от исходного параллелограмма треугольники равной площади.

22. Через точку $P$ проведены отрезки $E F, K L$ и $M N$, параллельные сторонам треугольника $A B C$ (рис.16). Докажите равновеликость треугольников $K E N$ и $M L F$.

23. В треугольник $A B C$ вписана окружность с центром $I$, которая касается его сторон $B C, C A$ и $A B$ в точках $A_{1}, B_{1}$ и $C_{1}$ соответственно. Отрезки $A I, B I$ и $C I$ пересекают окружность в точках $A_{2}$, $B_{2}$ и $C_{2}$. Докажите, что площадь треугольника $A_{2} B_{2} C_{2}$ равна половине площади шестиугольника $B_{1} A_{2} C_{1} B_{2} A_{1} C_{2}$.

24. В выпуклом четырехугольнике провели отрезки, соединяющие середины противоположных сторон. Их точку пересечения соединили с вершинами четырехугольника. Докажите, что суммы площадей противолежащих треугольников равны.

\section{Почему обжигает пар?}

\section{Л.АШКИНАЗИ}

\begin{abstract}
$\mathrm{B}$ О МНОГИХ ЗАДАЧНИКАХ МОЖно встретить вопрос - «почему обжигает пар» или «почему пар обжигает сильнее, чем вода той же температуры». И дается ответ - «потому, что происходит конденсация с тепловыделением». Но, во-первых, не всякое тепловыделение заметно поднимает температуру; нужно хотя бы простейшей оценкой показать, что эффект заметен. Вовторых, вдумчивый школьник может выдви-
\end{abstract}

DOI: https://doi.org/10.4213/kvant20200905 нуть такое возражение - горячий металл обжигает сильнее горячего дерева, а холодный металл «холодит» сильнее камня, т.е. тепловые потоки зависят от теплопроводности. Пар - это газ, его теплопроводность много меньше, чем у жидкости, так что все должно быть наоборот.

Теплопроводность пара действительно мала, однако в целом это возражение неправильное. В этом нам еще предстоит разобраться, а начнем мы с понятия теплопроводности, которое употребляем интуитивно. Мы введем его для частного случая, но применяется оно шире; при этом из нашего частного вывода станет понятно ограничение, при котором можно пользоваться этим понятием.

Запишем формулу

$$
Q=c m \Delta T,
$$

которая вам известна. Здесь при чтении надо сделать короткую паузу - вспомнить, что как обозначается, написать на бумажке раз- 